\title{
Commentary Response for "LARC Provision by Family Physicians: Low But on the Rise"
}

\author{
Marji Gold, MD
}

\section{(J Am Board Fam Med 2019;32:4-5.)}

Family physicians (FPs) are well poised to offer comprehensive contraceptive services to their patients, since they see patients across the life cycle and often provide maternity care and many preventative health services, including Papanicolaou smears and sexually transmitted infections (STI) screening. In addition, since they are often the only clinicians available in many parts of the country, their provision of contraceptive services can greatly improve timely access to this care.

This short article, "Long-Acting Reversible Contraception (LARC) Provision by Family Physicians: Low But on the Rise," analyzed data collected by the ABFM to document the small percentage of FPs who are actually providing LARC services (intrauterine device [IUD] and contraceptive implant insertion and removal) in their practices.

The authors point out that there are 2 overarching issues to address to increase access to LARC care in family medicine: 1) increased training in family medicine residency programs, and 2) systems changes in practices and reimbursement policies that allow trained clinicians to actually offer services. While focusing on these changes, it is important to recognize the difference between increasing access to LARC devices and increasing patient use of these methods.

Training to increase the number of FPs who have the skills to provide LARC care, to improve access to patients who want to use these methods, should include enhanced patient-centered, non-

From the Department of Family and Social Medicine, Montefiore Medical Center/Albert Einstein School of Medicine, Bronx, NY.

Funding: none.

Conflict of interest: none declared.

Corresponding author: Marji Gold, MD, Department of Family and Social Medicine, Montefiore Medical Center/ Albert Einstein School of Medicine, Bronx, NY 10467 (E-mail: marji.Gold@einstein.yu.edu). judgmental counseling, procedural skills for insertion and removal, and knowledge of systembased practice so trained FP's can overcome barriers to implementing LARC services in their practice.

Training should be offered within a reproductive justice framework. This intersectional approach highlights the impact of the long history of reproductive coercion and abuse of patients, especially of poor women of color, ${ }^{1,2}$ by the medical profession, and stresses the importance of personal bodily autonomy and each person's right to decide whether (and when) to have children, or not. FPs must be aware of this history, and how the power and role of our profession impacts patient trust and the doctor-patient relationship.

Although LARC devices are the most effective methods to prevent pregnancy, not all patients want a method that is inserted in their body, and that may require a clinician for removal should they choose to discontinue the method. Clinicians who are trained in LARC insertion often use "tiered counseling" to present the most effective methods first, rather than using a patient-centered approach and inquiring what the patient's needs and preferences are about pregnancy prevention and contraceptive options. Clinicians often feel they have "failed" if a patient leaves their office without a LARC device. Many measures of quality care include the percent of patients in a practice who have received LARC devices, rather than ensuring access to whatever method the patient prefers, or no method at all. Focusing on a patient-centered approach to counseling and service delivery can ensure that FPs are not repeating the mistakes of our predecessors, and are working toward more equitable care delivery.

This article stresses the importance of including routine training in LARC provision in family med- 
icine residency programs as a necessary step toward increasing access. To increase access by trained clinicians, the authors also discuss the importance of addressing systemic barriers, such as the cost of and reimbursement for the devices, and changing the clinic work flow to include longer time slots for scheduling procedures. An emphasis on increasing medical education in LARC procedures must also include the history of LARC use and abuse in this country, as well as patient-centered counseling and shared decision making. Training must be seen not as a way to increase LARC use, but as a means to improve comprehensive contraception access and enhance patient autonomy.

To see this article online, please go to: http://jabfm.org/content/ 32/1/4.full.

\section{References}

1. Roberts D. Killing the black body: Race, reproduction, and the meaning of liberty. New York, NY: Knopf Doubleday; 1997 (reprinted 2017).

2. Ross LJ, Solinger R. Reproductive justice. Oakland, CA: University of California Press; 2017. 